\title{
A Cumulant-Based Characterization of the Aggregate Interference Power in Wireless Networks
}

\author{
Muhammad Aljuaid and Halim Yanikomeroglu \\ Department of Systems and Computer Engineering \\ Carleton University \\ Ottawa, Canada \\ Email: \{majuaid, halim\}@sce.carleton.ca
}

\begin{abstract}
The importance of characterizing the aggregate interference power generated by a wireless network has increased with the emergence of different types of wireless networks such as ad-hoc networks, sensor networks, and cognitive radios. A cumulant-based characterization of this aggregate interference is an attractive approach. A number or recent papers in literature have dealt with cumulants of the aggregate interference but under specific scenarios. In this paper, we introduce a simple yet comprehensive method to determine the cumulants of the aggregate interference power originating from a wireless network. This method is quite general and applicable for finite and infinite network sizes, and it is flexible to encompass different system and propagation parameters such as large-scale fading, small-scale fading or even composite fading. We also investigate the behavior of these cumulants with respect to changes in the network size and fading distributions.

Index Terms-Aggregate Interference, Cumulants, Fading, Poisson Point Process.
\end{abstract}

\section{INTRODUCTION}

The importance of characterizing the aggregate interference generated by a wireless network has some history dating back at least to packet radios [1]. This importance has increased with the emergence of different types of wireless networks over the past two decades, e.g., CDMA [2], ad-hoc and sensor networks [3], spectrum sharing and cognitive radio networks [4]-[6]. Further history and references are provided in [7], [8].

Many papers investigate the characterization of the aggregate interference power by modeling the wireless network as a Poisson field of independent interferers. They reach to closedform expressions for the characteristic function. However, the inversion of this characteristic function into a closed-form probability density function (PDF) or cumulative distribution function (CDF) is not achievable, except for a few cases. A viable option to overcome this inversion problem is to obtain moments (or cumulants) of the aggregate interference and then to apply moments-based (or cumulants-based) approximations or bounds [2], [4]-[6], [9], [10].

While the moments and cumulants are closely related, cumulants have some properties making them more attractive for characterizing the aggregate interference of a Poisson field of interferers. Among these properties is that the $m$ th cumulant of the sum of independent random variables is equal to the

This work was funded by Saudi Aramco, Dhahran, Saudi Arabia. sum of the individual $m$ th cumulants [11]. Combining this property with the independence property of disjoint Poisson fields provides a powerful tool in characterizing the aggregate interference in a wireless network.

To the best of our knowledge, few papers in literature have dealt with cumulants of the aggregate interference power. These papers focus on specific scenarios. For example, [4] and [5] deal with cumulants for non-fading scenarios; [2] provides an integral form to compute the cumulants for out-ofcell interference in a CDMA networks; and [6] considers an infinite field with an exclusion region. Extending these results and generalizing them for a wide range of scenarios are among the contributions of this paper.

We characterize the distribution of the aggregate interference using cumulants. We provide a very simple yet powerful method to determine the cumulants. The method is flexible enough to be applicable to a wide range of scenarios including, but not limited to, the following: finite fields, infinite fields, different fading distributions (e.g., Rayleigh, Rician, shadowing, generalized- $K$ ), and variations in power levels. Moreover, we investigate the behavior of cumulants, and hence the aggregate interference power, with respect to changes in the network size and for various fading distributions.

The rest of the paper is organized as follows. Section II describes the system model and provides some background information on cumulants. Section III presents a simple method to calculate the cumulants of the aggregate interference power. The effects of the network size and fading distributions on cumulants are investigated in Section IV and Section V, respectively. Section VI discusses the cumulants-based approximations of the distribution of the aggregate interference power. Finally, Section VII concludes the paper with some remarks.

\section{System Model And Statistical Preliminaries}

The analysis in this paper is based on modeling a wireless network as a two-dimensional field of interferers deployed over a region of area $A$ with an annular sector shape (ring or disk shapes are special cases). The set of active interfering transmitters (nodes) is assumed to follow a Poisson point process with a homogeneous density $\lambda$. Based on this assumption, the number of interferers in a region is a Poisson random 
variable with a parameter specified by the multiplication of $\lambda$ by the area of that region. Moreover, the number of interferers in disjoint regions are independent Poisson random variables [12]. The field of interferers is assumed to have an inner radius of $r_{o}$. We call exclusion region the disk $b\left(O, r_{o}\right)$ of radius $r_{o}$ and centered at the origin. The field has a radial depth of $L$, making the outer radius of the field $r_{o}+L$. The field spans over an angle of $\theta$ as seen by the victim receiver at the origin, as illustrated in Fig. 1.

The individual interference power received by a victim receiver at the origin due to the transmission of node $i$ is denoted by $I_{i}$. Under the assumption of incoherent addition of interfering signals, the aggregate interference power received by the victim receiver can be expressed as

$$
I_{A}=\sum_{i \in \Lambda} I_{i}=\sum_{i \in \Lambda} X_{i} g\left(r_{i}\right)
$$

where $\Lambda$ is a set of interfering nodes, $X_{i}$ is a positive random variable that can be modeled as the multiplication of deterministic quantities and various random variables reflecting the transmit power, antenna gain, channel attenuation (including multipath and shadow fading) and other factors. In analyzing the aggregate interference of a Poisson field, it is common to assume that $X_{i}$ s are independent and identically distributed (i.i.d.) random variables [4], [6], [7], [13]. In this paper, we follow the same assumption. The function $g\left(r_{i}\right)$ represents a path-loss model (or more precisely the distance-dependent attenuation), discussed in the following subsection.

\section{A. Path-Loss Models}

The most common path-loss model used in literature is

$$
g\left(r_{i}\right)=k r_{i}^{-n}, r_{i} \geq 0,
$$

where $k$ is a constant, $r_{i}$ is the distance between the interfering node $i$ and the victim receiver, and $n$ is the path-loss exponent. This model is commonly used in contexts similar to the one we consider in this paper due to its mathematical tractability [14]. However, this model suffers from a singularity at $r_{i}=0$. Therefore, it is known as a singular (or unbounded) path-loss model [14], [15].

There are non-singular (bounded) path-loss models used in literature as well, e.g., [6], [10], [15], [16]. These nonsingular models are similar, with some variations. Following is an example:

$$
g\left(r_{i}\right)=\left\{\begin{array}{ll}
k r_{i}^{-n}, & r_{i} \geq r_{c} \\
k r_{c}^{-n}, & r_{i}<r_{c}
\end{array},\right.
$$

where $r_{c}>0$ is the radius at which the slope of the model starts changing.

It is indicated in [15] that the selection of the path-loss model has a significant impact on the characterization of the aggregate interference. Authors in [14] investigated the effect of the unbounded model on the performance analysis of wireless networks and indicated that more realistic performance figures are obtained by using bounded models. We therefore consider the use of a non-singular model, specifically (3) with $n>2$. Without loss of generality, we take $k=1$ assuming its effect is absorbed by $X_{i}$.

\section{B. Statistical Preliminaries on Cumulants}

The $m$ th cumulant of a random variable $X$ whose characteristic function is $\phi_{X}(w)$ can be obtained by [11]

$$
\kappa_{m}=\frac{1}{j^{m}}\left[\frac{d^{m} \ln (\phi(\omega))}{d \omega^{m}}\right]_{\omega=0} .
$$

Cumulants have some properties that make them more attractive for the problem considered in this paper. Among these properties are the following:

- The $m$ th cumulant of the sum of two independent random variables is equal to the sum of the individual $m$ th cumulants of these independent random variables [11].

- If $Y=\frac{X-\tilde{\mu}}{\sigma}$ where $\tilde{\mu}$ and $\sigma$ are the mean and the standard deviation of the random variable $X$ respectively, then $\kappa_{1}(Y)=0$, and $\kappa_{m}(Y)=\kappa_{m}(X) \sigma^{-m}=\kappa_{m}(X) \kappa_{2}(X)^{-m / 2}$ for $m \geq 2$ [17] where $\kappa_{m}(Y)$ denotes the $m$ th cumulant of $Y$.

- Cumulants can be used to obtain some important measures of the distribution, like mean $\left(\kappa_{1}\right)$, variance $\left(\kappa_{2}\right)$, skewness $\left(\kappa_{3} \kappa_{2}^{-3 / 2}\right)$ and kurtosis excess $\left(\kappa_{4} \kappa_{2}^{-2}\right)$ [17].

- The cumulants of a random variable are closely related to its moments. For example, $\kappa_{1}=\tilde{\mu}_{1}, \kappa_{2}=\mu_{2}=\sigma^{2}$, $\kappa_{3}=\mu_{3}$, and $\kappa_{4}=\mu_{4}-3 \mu_{2}^{2}$ where $\tilde{\mu}_{m}$ is the $m$ th raw moment, $\mu_{m}$ is $m$ th central moment, and $\sigma^{2}$ is the variance. General expressions relating cumulants to central or raw moments are provided in [17].

- Cumulants of a random variable can be used to approximate the distribution function of that random variable through some approaches like an Edgeworth series expansion [2]. Alternatively, cumulants can be used to determine the moments, and then moments-based approximations can be applied [17].

\section{Cumulants of $I_{A}$}

The main contribution of this paper is stated in the following proposition. To value this proposition, it is helpful to imagine that the field of interferers would virtually collapse to a subfield with an effective area $A_{\text {eff }}$, an inner radius $r_{o}$, and an outer radius $r_{\text {eff }}$. The average number of interfering nodes within this subfield is $N_{\text {eff }}$.

Proposition 1: The $m$ th cumulant of the distribution of the aggregate interference power received by a victim receiver at the origin from an annulus-shaped Poisson field of i.i.d. interferers is

$$
\kappa_{m}\left(I_{A}\right)=N_{\text {eff }}(m) \tilde{\mu}_{m}\left(I_{r_{o}}\right),
$$

where $N_{\text {eff }}(m)$ is the average number of interfering nodes within a radius of $r_{\text {eff }}(m)$ from the victim receiver, $\tilde{\mu}_{m}\left(I_{r_{o}}\right)$ is the $m$ th raw moment of the distribution of the interference power received by the victim receiver from an interfering node at distance $r_{o}$. We then have

$$
N_{\text {eff }}(m)=\lambda A_{\text {eff }}(m),
$$


where

$$
A_{\text {eff }}(m)=\left\{b\left(O, r_{\text {eff }}(m)\right) \cap A\right\}=\frac{1}{2} \theta\left[r_{\text {eff }}^{2}(m)-r_{o}^{2}\right],
$$

and

$$
\begin{aligned}
r_{\mathrm{eff}}(m) & =\hat{r} \sqrt{1+\frac{2}{m n-2}\left(1-\left[\frac{\hat{r}}{r_{o}+L}\right]^{m n-2}\right)}, \\
\hat{r} & =\max \left(\min \left(r_{c}, r_{o}+L\right), r_{o}\right) .
\end{aligned}
$$

Note that $\tilde{\mu}_{m}\left(I_{r_{o}}\right)=\tilde{\mu}_{m}(X)\left[g\left(r_{o}\right)\right]^{m}$ where $\tilde{\mu}_{m}(X)$ is the $m$ th raw moment of $X_{i}$.

Proof: Using Campbell's theory for a Poisson Point Process [7], [12], it can be shown that:

$$
\phi_{I_{A}}(\omega)=\exp \left(-\theta \lambda \int_{r_{o}}^{r_{o}+L} \int_{0}^{\infty}\left[1-e^{j \omega x g(r)}\right] f_{X}(x) r d x d r\right) \text {, }
$$

where $\phi_{I_{A}}(\omega)$ is the characteristic function of $I_{A}, f_{X}(x)$ is the PDF of $X_{i}$, and $j=\sqrt{-1}$. Denoting the characteristic function of $X_{i}$ by $\phi_{X}(\omega)$, (9) can be rewritten as

$$
\phi_{I_{A}}(\omega)=\exp \left(-\theta \lambda \int_{r_{o}}^{r_{o}+L}\left[1-\phi_{X}(\omega g(r))\right] r d r\right) .
$$

From (4) and (10),

$$
\kappa_{m}\left(I_{A}\right)=\theta \lambda \tilde{\mu}_{m}(X) \int_{r_{o}}^{r_{o}+L} g^{m}(r) r d r .
$$

Then, the proof follows directly from (11) with some mathematical manipulations and arrangements.

Equation (5) is simple yet flexible in the sense that it can be applied to a wide range of scenarios such as finite fields, infinite fields (see Section IV), and to different fading distributions (see Section V). Moreover, (5) is applicable for many field's topologies, including when the victim receiver is in the middle of the field or away from it.

\section{EfFect of Spatial Size of the Field AND INTERFERERS DENSITY ON CUMULANTS}

The spatial size of the field is controlled by $L, r_{o}$, and $\theta$. The changes in $L$ affect $r_{\text {eff }}$, and hence $A_{\text {eff }}$ and $\kappa_{m}$. However, this effect is limited. As $L$ increases, $r_{\text {eff }}$ increases but it converges to a finite value regardless of the further increase in $L$. As $m$ or $n$ increases, this convergence occurs faster. The effect of changes in $L$ is significant only for lower-order cumulants and for $L$ closer to or less than $\max \left(r_{c}, r_{o}\right)$. As $L$ approaches the value of $\max \left(r_{c}, r_{o}\right)$, its effect becomes weaker and it will be negligible when $\left(\frac{\max \left(r_{c}, r_{o}\right)}{r_{o}+L}\right)^{m n-2} \ll 1$.

As an example, Fig. 2 shows that the convergence value for $\kappa_{1}$ is almost reached when $L>100 \mathrm{~m}$ for a field with no exclusion region $\left(r_{o}=0\right)$. However, $\kappa_{2}$ is almost at the convergence value when $L>2 \mathrm{~m}$. The higher-order cumulants converge faster (at lower values of $L$ ). Therefore, doubling $L$ or even expanding it to infinity has a negligible (or practically no) effect on $\kappa_{m}$ provided that $\kappa_{m}$ is almost at the convergence value.
If there is an exclusion region, e.g., $r_{o}=10 \mathrm{~m}$, then the convergence value will be almost reached when $L>1 \mathrm{Km}$ for the first cumulant and $L>20 \mathrm{~m}$ for the second cumulant. A smaller $L$ will be required to closely approach the convergence level for higher cumulants, as seen in Fig. 3.

Interestingly, the value of $r_{\text {eff }}$ converges to $\max \left(r_{c}, r_{o}\right) \sqrt{1+\frac{2}{m n-2}}$ as $L \rightarrow \infty$. From this, we may conclude that $\kappa_{m}$ is controlled mainly by the region which is close to the victim receiver. The dominant region for $\kappa_{1}$ (the average) is wider than that for $\kappa_{2}$ (the variance). It shrinks as $m$ increases. On the other hand, this dominant region expands as $r_{o}$ (the exclusion region) increases.

The exclusion region has an effect on $A_{\text {eff }}$ of an order of $r_{o}^{2}$ (provided that $r_{o}>r_{c}$ ). Moreover, $r_{o}$ affects the value of $\tilde{\mu}_{m}\left(I_{r_{o}}\right)$ by an order of $r_{o}^{-m n}$. Therefore, the net effect of $r_{o}$ on $\kappa_{m}$ is in the order of $r_{o}^{2-m n}$, which suggests that increasing the value of $r_{o}$ is an effective way to lower the aggregate interference. However, increasing $r_{o}$ may contradict the performance objectives of the wireless network [5], [18], [19]. Therefore, an optimal tradeoff is to be found.

Regarding the effect of the active node density $(\lambda)$, it has a linear effect on all cumulants. Therefore, it is one of the important parameters that could be used to control the level of interference at the victim receiver.

Similarly, $\theta$ has a linear effect on the cumulants. However, it is limited to the range $[0,2 \pi]$. It may reflect the effectiveness of using a directional antenna at the victim receiver.

The effect of the increase in the network size (spatial size and node density) on the average of the aggregate interference is considered in [3] and [20]. Moreover, the effect on the variance is discussed in [21]. However, to the best of our knowledge, this paper is the first to address the effect of the spatial size on all cumulants of the aggregate interference.

\section{EfFect of the Distribution of $X$ on Cumulants}

The random variable $X$ encompasses many system and channel parameters. It might be modeled as the multiplication of some deterministic and random variables reflecting the effect of different parameters, such as fluctuations in power level and antenna gains, multipath fading, and shadow fading ${ }^{1}$.

$$
X_{i}=\prod_{l} X_{i, l}
$$

where $X_{i, l}$ is a deterministic or a random variable. (A similar representation is used in [7].)

From (5) and $\tilde{\mu}_{m}\left(I_{r_{o}}\right)=\tilde{\mu}_{m}(X)\left[g\left(r_{o}\right)\right]^{m}$, it is clear that the distribution of $X_{i}$ has a major influence on $\kappa_{m}$, and hence the distribution of the aggregate interference power. A similar observation on the influence of the fading distribution, equivalently of the distribution of $X_{i}$, on the aggregate interference appears in [15].

Following are some examples of $\kappa_{m}$ under different distributions of $X_{i}$. While $X_{i}$ can be more general than just fading, examples given here consider fading distributions only.

\footnotetext{
${ }^{1}$ Equation (12) is general enough to account for the adjacent-channel interference. However, it is not discussed here due to space limitation.
} 


\section{A. Multipath Fading}

A general model that could be used to reflect the multipath fading on the interference power is the Gamma distribution (under the assumption of Nakagami fading for the interference signal). The PDF of the Gamma distribution is

$$
f_{X}(x)=\left(\frac{\nu}{\Omega}\right)^{\nu} \frac{x^{\nu-1}}{\Gamma(\nu)} e^{-\frac{\nu}{\Omega} x}, x>0, \nu \geq \frac{1}{2},
$$

where $\nu$ is the shape parameter, $\Gamma($.$) is the Gamma function,$ and $\Omega$ is the average power, i.e., $E[X]$, which is commonly assumed to be equal to unity [22]. The $m$ th raw moment of the Gamma distribution with $\Omega=1$ can be expressed as

$$
\tilde{\mu}_{m}(X)=\nu^{-m} \frac{\Gamma(\nu+m)}{\Gamma(\nu)} .
$$

Therefore,

$$
\kappa_{m}\left(I_{A}\right)=N_{\text {eff }}(m)\left[g\left(r_{o}\right)\right]^{m} \nu^{-m} \frac{\Gamma(\nu+m)}{\Gamma(\nu)} .
$$

The exponential distribution (corresponding to Rayleigh fading for the interference signal) is a special case of the Gamma distribution, when $\nu=1$.

\section{B. Shadow Fading}

Shadow fading is usually modeled by a lognormal random variable whose mean and standard deviation in the $\mathrm{dB}$ domain are zero and $\sigma_{\mathrm{dB}}$, respectively. Let $X_{i}=10^{S_{i} / 10}$, representing the shadowing effect in the linear domain, where $S_{i} \sim \mathcal{N}\left(0, \sigma_{\mathrm{dB}}^{2}\right)$. After a proper normalization of $X_{i}$, it can be shown that

$$
\tilde{\mu}_{m}(X)=e^{\frac{1}{2}\left(m \frac{\ln 10}{10} \sigma_{\mathrm{dB}}\right)^{2}}
$$

and

$$
\kappa_{m}\left(I_{A}\right)=N_{\text {eff }}(m)\left[g\left(r_{o}\right)\right]^{m} e^{\frac{1}{2}\left(m \frac{\ln 10}{10} \sigma_{\mathrm{dB}}\right)^{2}} .
$$

\section{Composite Fading}

If the wireless channel suffers from multipath and shadow fading simultaneously, $X_{i}$ can be modeled as the product of two independent random variables [7]. Assuming these random variables are Gamma (for multipath) and lognormal (for shadowing), the cumulants of the normalized aggregate interference can be expressed as

$$
\kappa_{m}\left(I_{A}\right)=N_{\mathrm{eff}}(m)\left[g\left(r_{o}\right)\right]^{m} e^{\frac{1}{2}\left(m \frac{\ln 10}{10} \sigma_{\mathrm{dB}}\right)^{2}} \nu^{-m} \frac{\Gamma(\nu+m)}{\Gamma(\nu)} .
$$

\section{Vi. Cumulants-based Approximations of the DISTRIBUTION OF $I_{A}$}

The distribution of $I_{A}$ can be approximated using a finite set of its cumulants. For example, an Edgeworth expansion is used in [2], [4], and [6] to approximate the PDF of the aggregate interference. The Edgeworth expansion is a simple approach used to expand the logarithm of the characteristic function using its Taylor series expansion. Based on this expansion and knowing the cumulants of a random variable, the PDF of this random variable can be approximated [2]. A detailed discussion of the Edgeworth expansion for approximating a PDF is provided in [17].

It is important to highlight that while the Edgeworth series expansion is an asymptotic expansion for the PDF, the finite Edgeworth series should be applied with some caution: it is applicable for moderately-skewed distributions only. Conditions under which the Edgeworth finite approximation can be used are discussed in [23].

If the distribution of the aggregate interference has a positive skewness, $I_{A}$ can be approximated by a lognormal random variable. An enhanced version of the lognormal approximation, called shifted lognormal approximation, is proposed in [24], and used in [6] to approximate the distribution of the aggregate interference power. The shifted lognormal approximation is a three-parameter approximation. These parameters are obtained from the first three cumulants. Figure 4 shows an example of approximating the PDF of $I_{A}$ by an Edgeworth series expansion and a shifted lognormal PDF. A Gaussian PDF is included in the figure as a reference.

As an alternative, cumulants can be used to calculate the moments. Then, moments-based approximations or bounds can be applied to approximate the distribution of $I_{A}$ [17].

\section{CONCLUSIONS}

In this work, we characterized the aggregate interference power generated by a wireless network through the approach of cumulants. We introduced a simple yet comprehensive method to calculate the cumulants. Our method is applicable for finite and infinite networks, and is flexible to encompass different system and propagation parameters including largescale fading, small-scale fading, and composite fading. We also discussed the behavior of these cumulants with respect to changes in the network size and fading distributions. Moreover, we highlighted some cumulants-based approximations of the distribution of the aggregate interference power.

\section{ACKNOWLEDGMENT}

The authors would like to thank Saad Al-Ahmadi and Sebastian Szyszkowicz for their helpful comments.

\section{REFERENCES}

[1] E. S. Sousa and J. A. Silvester, "Optimum transmission ranges in a direct-sequence spread-spectrum multihop packet radio network," IEEE J. Sel. Areas Commun., vol. 8, no. 5, pp. 762-771, June 1990.

[2] C. C. Chan and S. V. Hanly, "Calculating the outage probability in a CDMA network with spatial Poisson traffic," IEEE Trans. Veh. Technol., vol. 50, no. 1, pp. 183-204, Jan. 2001.

[3] M. Haenggi, "On distances in uniformly random networks," IEEE Trans. Inf. Theory, vol. 51, no. 10, pp. 3584-3586, Oct. 2005.

[4] R. Menon, R. M. Buehrer, and J. H. Reed, "Outage probability based comparison of underlay and overlay spectrum sharing techniques," in Proc. 1st IEEE Symp. Dynamic Spectrum Access Networks (DySPAN'05), Baltimore, MD, USA, Nov. 2005, pp. 101-109.

[5] — , "Impact of exclusion region and spreading in spectrum-sharing ad hoc networks," in Proc. First Internation Workshop Technology and Policy for Accessing Spectrum, Boston, MA, USA, Aug. 2006.

[6] A. Ghasemi and E. S. Sousa, "Interference aggregation in spectrumsensing cognitive wireless networks," IEEE J. Sel. Topics Signal Process., vol. 2, no. 1, pp. 41-56, Feb. 2008.

[7] M. Z. Win, P. C. Pinto, and L. A. Shepp, "A mathematical theory of network interference and its applications," Proceedings of the IEEE, vol. 97, no. 2, pp. 205-230, Feb. 2009. 


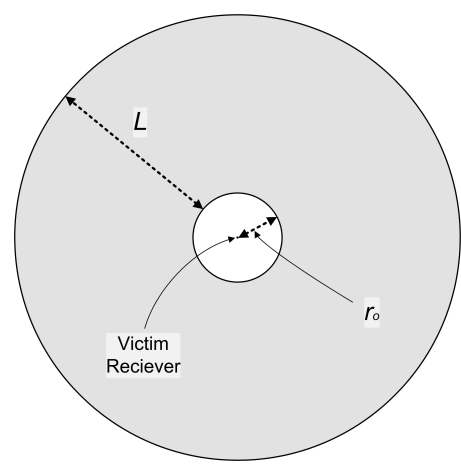

Fig. 1. Interferers field layout $(\theta=2 \pi)$.

[8] M. Haenggi, J. G. Andrews, F. Baccelli, O. Dousse, and M. Franceschetti, "Stochastic geometry and random graphs for the analysis and design of wireless networks," IEEE J. Sel. Areas Commun., vol. 27, no. 7, pp. 1029-1046, Sep. 2009.

[9] S. Weber, J. Andrews, and N. Jindal, "The effect of fading, channel inversion, and threshold scheduling on ad hoc networks," IEEE Trans. Inf. Theory, vol. 53, no. 11, pp. 4127-4149, Nov. 2007.

[10] E. Salbaroli and A. Zanella, "Interference analysis in a Poisson field of nodes of finite area," IEEE Trans. Veh. Technol., vol. 58, no. 4, pp. 1776-1783, May 2009.

[11] A. Hyvarinen, J. Karhunen, and E. Oja, Independent Component Analysis. New York, USA: Wiley, 2001.

[12] J. F. C. Kingman, Poisson Processes. New York: Oxford University Press, 1993

[13] E. S. Sousa, "Perfromance of a spread spectrum packet radio network link in a Poisson field of interferers," IEEE Trans. Inf. Theory, vol. 38, no. 6, pp. 1743-1754, Nov. 1992.

[14] H. Inaltekin, M. Chiang, H. V. Poor, and S. B. Wicker, "On unbounded path-loss models: effects of singularity on wireless network perfomance," IEEE J. Sel. Areas Commun., vol. 27, no. 7, pp. 10781091, Sep. 2009.

[15] R. K. Ganti and M. Haenggi, "Interference in ad hoc networks with general motion-invariant node distribution," in Proc. IEEE International Symposium on Information Theory (ISIT'08), Toronto, Canada, Jul. 2008.

[16] J. W. Gluck and E. Geraniotis, "Throughput and packet error probability in cellular frequency-hopped spread spectrum radio networks," IEEE J. Sel. Areas Commun., vol. 7, no. 1, pp. 148-160, Jan. 1989.

[17] A. Stuart and J. K. Ord, Kendall's Advanced Theory of Statistics, 6th ed. London, UK: Edward Arnold, 1994.

[18] A. Hasan and J. G. Andrews, "The guard zone in wireless ad hoc networks," IEEE Trans. Wireless Commun., vol. 6, no. 3, pp. 897-906, Mar. 2007.

[19] Y. M. Shobowale and K. A. Hamdi, "A unified model for interference analysis in unlicensed frequency bands," IEEE Trans. Wireless Commun., vol. 8, no. 8, pp. 4004-4013, Aug. 2009.

[20] M. Aljuaid and H. Yanikomeroglu, "On the asymptotic analysis of average interference power generated by a wireless sensor network," in Proc. IEEE Vehicular Technology Conference (VTC) 2008-Fall, Calgary, AB, Canada, Sep. 2008

[21] — " "Impact of secondary users field size on spectrum sharing opportunities," in Proc. IEEE Wireless Communications and Networking Conference (WCNC) 2010, Sydney, Australia, Apr. 2010.

[22] P. M. Shankar, "Perfromance analysis of diversity combining algorithms in shadowed fading channels," Wireless Personal Communications, vol. 37, no. 1-2, pp. 61-72, Apr. 2006.

[23] D. E. Barton and K. E. Dennis, "The conditions under which GramCharlier and Edgeworth curves are positive definite and unimodal," Biometrika, vol. 39, 1952.

[24] K. L. Q. Read, "A lognormal approximation for the collector's problem," The American Statistcian, vol. 52, no. 2, pp. 175-180, May 1998.

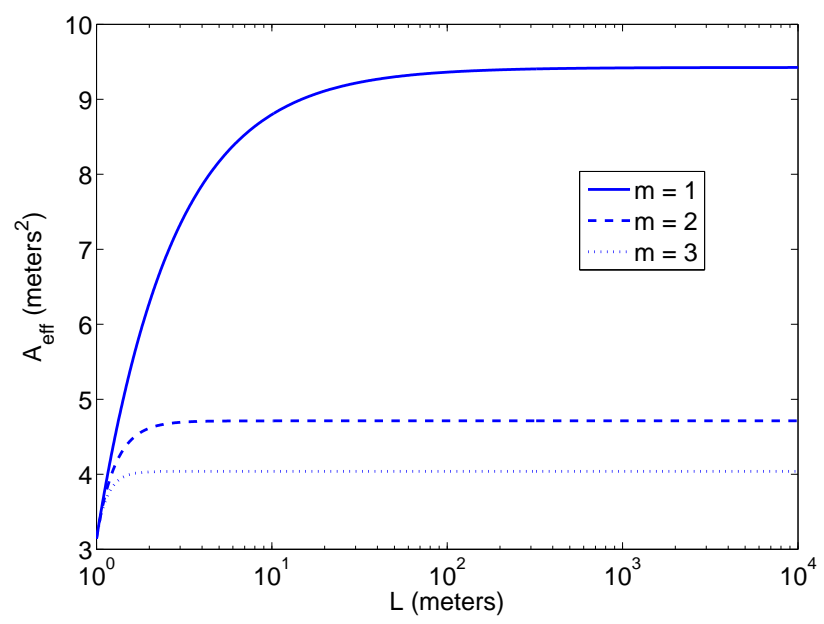

Fig. 2. The effect of $L$ on $A_{\text {eff }}$, and hence on $\kappa_{m}$ for the case of no exclusion region $\left(r_{o}=0\right)$ around the victim receiver $\left(r_{c}=1\right.$ meter, $\theta=2 \pi$ and $n=3$ )

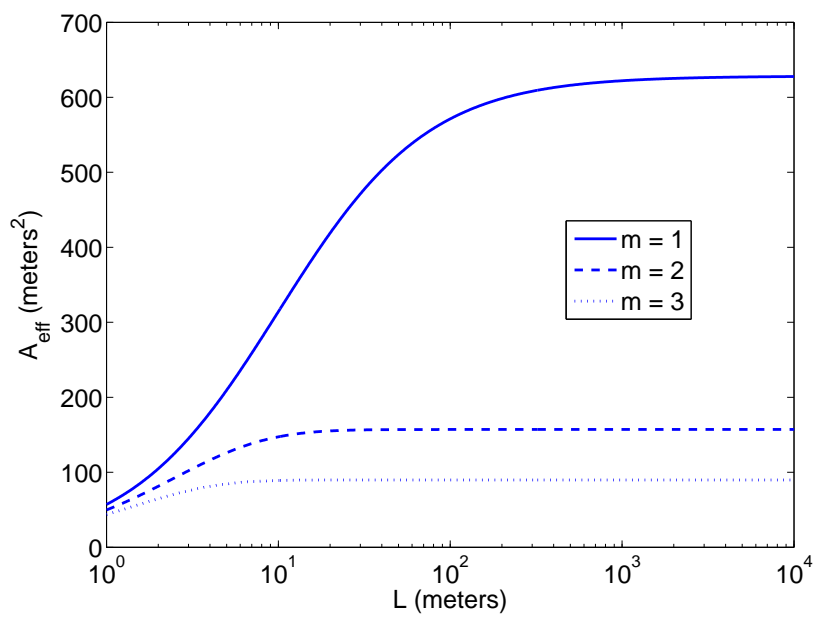

Fig. 3. The effect of $L$ on $A_{\text {eff }}$, and hence on $\kappa_{m}$ for the case of an exclusion region of $r_{o}=10$ meters $\left(r_{c}=1\right.$ meter, $\theta=2 \pi$ and $\left.n=3\right)$.

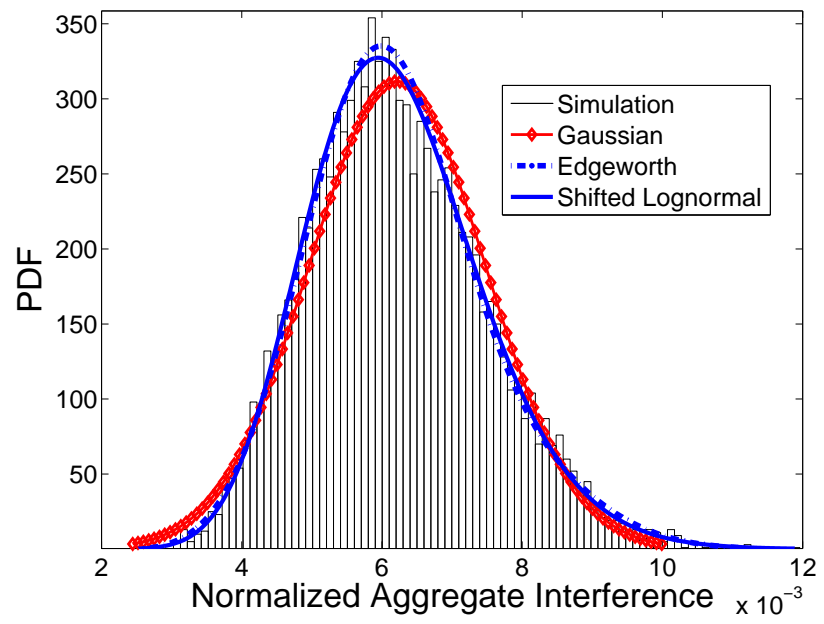

Fig. 4. The Monte Carlo simulation and different approximations of the PDF of $I_{A}\left(r_{o}=10\right.$ meters, $L=1000$ meters, $r_{c}=1$ meter, $\theta=2 \pi$ and $n=3)$. 